\title{
Potential of mean force between two nanometer-scale particles in a polymer solution
}

\author{
M. Doxastakis, Y.-L. Chen, and J. J. de Pablo ${ }^{a)}$ \\ Department of Chemical and Biological Engineering, University of Wisconsin, Madison, \\ Wisconsin 53706-1691
}

(Received 23 February 2005; accepted 20 May 2005; published online 22 July 2005)

\begin{abstract}
Expanded ensemble density-of-states simulations and a connectivity altering algorithm are used to investigate the effective interactions that arise between nanoparticles suspended in polymer solutions. Our calculations with systems of long polymeric chains reveal oscillations in the effective polymer-induced interactions between the particles, even at low concentrations. The range of these interactions is considerably longer than originally anticipated, and their origin is traced back to the chain-end effects and density fluctuations that were absent in previous treatments of these systems. (C) 2005 American Institute of Physics. [DOI: 10.1063/1.1953575]
\end{abstract}

\section{INTRODUCTION}

Depletion interactions between colloids induced by the presence of macromolecules have been studied by a variety of different approaches, ranging from theoretical studies and computer simulations to experimental investigations. Asakura and Oosawa ${ }^{1,2}$ first showed analytically that colloids in a polymer bath exhibit an attractive depletion interaction with a range approximately two times the radius of gyration $\left(R_{g}\right)$ of the macromolecules. Their simple model describes well the dilute regime in which the polymers are small relative to the colloid. Mean-field theory has also examined the effects of nondilute polymer concentrations in the semidilute regime where macromolecules start to interact and overlap each other. ${ }^{3}$ Several theoretical studies have followed, in pursuit of a complete description of the physics underlying the polymer-induced interactions between the colloids. ${ }^{4-16}$ Integral equation theory was first used by Yethiraj et al. ${ }^{17}$ to examine the polymer-induced potential between two large colloids and, more recently, by Schweizer and co-workers ${ }^{18-22}$ to investigate the depletion attraction and phase behavior of nondilute colloid-polymer mixtures. Advanced experimental techniques have been employed to resolve and measure the magnitude of such interactions, ${ }^{21,23-26}$ but interpreting the results using simple theoretical models has not been straightforward. ${ }^{27}$

Simulations offer a useful tool to study the physics of the colloid-polymer mixtures, provided that the methods and the model can accurately describe the system at the necessary level of detail. Early computations by Meijer and Frenkel addressed the equation of state and the phase diagram of a simple model. ${ }^{28}$ A Monte Carlo simulation with the fluctuating bond lattice model provided insight into the potential of mean force (PMF) between two particles immersed in a polymer solution. ${ }^{29}$ Bolhuis and co-workers presented a systematic study of the thermodynamics of colloid-polymer mixtures using Monte Carlo simulations. ${ }^{30-33}$ Polymers were represented as "soft colloids" in the limit where the chain

${ }^{a)}$ Electronic mail: depablo@engr.wisc.edu dimensions are small relative to the hard-sphere particles. In the opposite "protein" limit, where the polymers are much larger than the particles, the macromolecules were simulated as self-avoiding walks on a cubic lattice.

We have recently performed Monte Carlo (MC) simulations of one small particle in a solution of bead-spring macromolecules of various sizes to address the protein limit without the restrictions imposed by the use of a lattice model. ${ }^{34}$ The colloid was represented as a sphere smaller than the chain size, but larger than the segment size. Under these conditions, the internal degrees of freedom of the segments of the chain in a continuum must be explicitly accounted for, but the actual chemical structure of the monomers can be omitted, thereby greatly simplifying the model. By applying advanced Monte Carlo moves such as double rebridging, ${ }^{35,36}$ it was possible to equilibrate long polymers, which were shown to exhibit a tendency to wrap around the particle. In the proximity of the colloid, polymer segments acquire preferential orientations and deform relative to their equilibrium size. Furthermore, an increased concentration of chain ends in the vicinity of the particle is evident at all densities - an effect that increases with chain length. Describing such effects through theoretical models is not straightforward, but as shown in this work, their impact on the polymer-induced interactions between small particles can be significant.

In this paper, we extend our previous simulations to the case of two particles in a polymer solution. The aim is to study the induced interactions in the "protein limit" at the crossover from the dilute to the semidilute polymer regime. The colloid density is always kept at the limit of infinite dilution. We couple a new powerful simulation technique, the expanded ensemble density of states (EXEDOS), ${ }^{37-39}$ with connectivity-altering algorithms for the polymers. This new methodology is capable of efficiently sampling the configuration space of the protein limit with the highest accuracy currently feasible. Through exhaustive sampling of polymer configurations, it is possible to extract the potential of mean force of two small colloidal particles in a solution of long 
macromolecules with an unprecedented level of precision. Results are compared to predictions of the polymer reference interaction site model with the Percus-Yevick Closure (PRISM-PY), which has been shown to capture qualitative aspects of the polymer-induced depletion attraction in polymer-dilute particle mixtures. ${ }^{18,21,34}$ The results of our simulations reveal that the range of polymer-induced interactions is much longer than anticipated in previous studies, raising questions about the meaning of a "dilute solution" in the context of nanoparticle suspensions.

\section{METHODOLOGY}

Polymers are modeled as bead-spring chains with repulsive Lennard-Jones interactions, truncated and shifted at the minimum,

$$
\begin{aligned}
U_{n b}(r)= & 4 \varepsilon\left[\left(\frac{\sigma}{r-R_{r}}\right)^{12}-\left(\frac{\sigma}{r-R_{r}}\right)^{6}\right]+\varepsilon, \\
& r-R_{r}<2^{1 / 6} \sigma, \\
U_{n b}(r)= & 0, \quad r-R_{r} \geqslant 2^{1 / 6} \sigma,
\end{aligned}
$$

where $R_{r}=0$ for monomer-monomer interactions. The bonding energy between consecutive monomers in a polymer chain is of harmonic form,

$$
U_{b}(r)=\frac{1}{2} k_{h}\left(r-2^{1 / 6} \sigma\right)^{2},
$$

with the elastic constant $k_{h}=10^{4}$. Two colloids are placed in each simulation system. Using Eq. (1) and a value of $R_{r}$, the interaction between each colloid and the monomers is taken into account from the surface of the particle. ${ }^{34}$

All systems are simulated at a temperature that makes the Barker-Henderson mean collision diameters ${ }^{40}$

$$
d(\beta)=-\int_{0}^{\infty}\left(e^{-\beta u_{n b}(r)}-1\right) d r
$$

equal to $\sigma$ for the nonbonded potential. This special temperature is given by $1 / \beta=k T=1.50579 \varepsilon$. This facilitates comparison of our results to available findings for hard spheres with corresponding diameters $d_{m}=\sigma$ for the monomer and $d_{c}=\sigma+2 R_{r}$ for the colloids.

As reported in Ref. 34, a series of off-lattice canonical MC simulations are conducted using a combination of trial moves to efficiently explore configuration space. In the case of long polymer chains, the most effective move for polymer end-to-end relaxation is the "double rebridging," a chain connectivity altering move $\mathrm{e}^{35,36}$ that rearranges two neighboring chains by exchanging parts of them. To demonstrate the importance of such connectivity altering moves for our systems, Fig. 1 illustrates the change imposed in the vicinity of the two colloids by the acceptance of a double rebridging move. In mixtures of colloids with long macromolecules, the polymer can wrap around the particles; efficient, polymerspecific algorithms are essential to ensure exhaustive sampling of configuration space and to minimize any inherent statistical errors.

To further enhance the quality of the calculated PMF, the so-called EXEDOS technique was also implemented. ${ }^{37-39}$
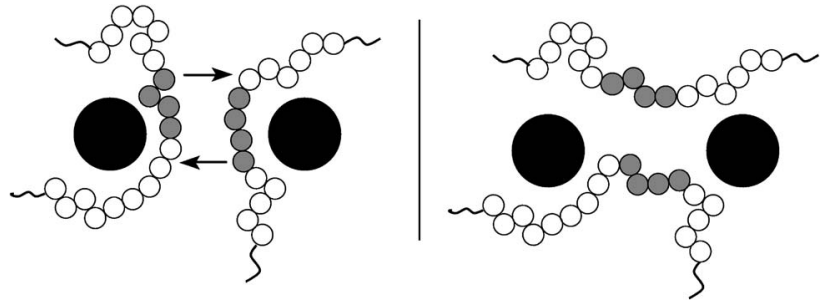

FIG. 1. Double rebridging move in the vicinity of two colloids. Bridges of two to four particles are performed at specific positions along the chains. The algorithm employed in this work preserves the monodispersity of the polymer solution.

The method relies on an extension of the density-of-states (DOS) algorithm first proposed by Wang and Landau. ${ }^{41}$ It permits uniform, non-Boltzmann sampling of the system along a specified reaction coordinate (the distance $x$ between the two particles in our systems). In contrast with umbrella sampling, the weight factors introduced to achieve a random walk over this reaction coordinate are calculated on the fly, in an iterative fashion. In EXEDOS, the distance between the two particles (the reaction coordinate $x$ ) is discretized into a number of bins; a histogram entry $h\left(x_{i}\right)$ and a statistical weight $g\left(x_{i}\right)$ are associated with bin $i$, corresponding to $x_{i}$. For simplicity, $g\left(x_{i}\right)$ is assumed to be uniform at the beginning of a simulation. Trial MC moves consist of attempted particle displacements along the reaction coordinate. Successive MC steps produce visits to distinct bins; every time that a bin is visited, its weight is multiplied by a convergence factor $f: g\left(x_{i}\right) \rightarrow f g\left(x_{i}\right) \quad\left[\right.$ or $\left.\ln g\left(x_{i}\right) \rightarrow \ln g\left(x_{i}\right)+\ln f\right]$. In the first "cycle" of the simulation, the value of $f(>1)$ is relatively large [e.g., $f=\exp (1)]$. That value of $f$ remains unchanged throughout the first cycle until the accumulated histogram of visited states $\left[h\left(x_{i}\right)\right]$ is sufficiently flat; a new simulation cycle is then started, with a lower value of $f$, e.g., $f_{\text {new }}=\sqrt{f_{\text {old }}}$. The histogram $h\left(x_{i}\right)$ is erased. Detailed balance is satisfied in the limit where $f$ approaches unity. ${ }^{41}$ Trial MC moves are accepted with probability

$$
\begin{aligned}
& P_{\text {acc }}(\text { old } \rightarrow \text { new }) \\
& \quad=\min \left\{1, \exp [-\beta \Delta U] \frac{x_{\text {old }}^{2}}{x_{\text {new }}^{2}} \exp [-\Delta \ln g]\right\} .
\end{aligned}
$$

Monte Carlo trial moves for the polymer molecules do not alter the separation $x$ between the two particles and are accepted according to the standard Boltzmann sampling criteria. A colloid displacement promotes a change in the reaction coordinate and its acceptance is weighted by the accumulated $g(x)$ corresponding to the specific $x$. The simulation has converged when, through consecutive cycles, $\ln f$ has decreased to a small preset value (e.g., $f=1+10^{-8}$ ). At that point, the density of states of each bin is not significantly altered and detailed balance is recovered. A more detailed description of the method and its application to nematic liquid crystals and proteins can be found in Refs. 37 and 42.

We performed simulations using two colloids of diameter $d_{c}=4.9 \sigma$. Polymer chains of length $N=16,120$, and 500 were studied in large boxes of 20000,30240 , and 50000 (for the longest chains) monomers. For each chain length the 
simulation box has to be greater than $4 R_{g}$ to avoid finite-size effects; as discussed later, the system must be at least onehalf box greater than the range of the "bridging forces" defined later in this work. The ratios $q=R_{g} / R$ of the chain to the colloid size are approximately 1, 3.6, and 8.56 for the three polymer lengths considered here. A range of polymer concentrations was examined for each polymer size, encompassing the dilute regime and the semidilute regime where chains begin to overlap. The range of the polymer-induced interaction between the colloids is expected to be greatly reduced above the crossover regime. ${ }^{43,44}$ We have discretized the separation of the two particles into small bins (of size always less than $0.05 \sigma$ ), and calculated each bin's weight factor required to achieve uniform sampling. A histogram was considered "flat" whenever the minimum population was at least $85 \%$ of the average. ${ }^{41}$ In some simulations (for extremely dilute systems), where increased accuracy was required, a 95\% flatness criterion was applied. All computations were completed with values for the logarithm of the convergence factor $f$ less than $10^{-8}$. Multiple simulations are performed in parallel (three machines for $N=16$ to ten machines for $N=500$ ), each with a different range of separation (different "windows"). Special care must be exercised to avoid boundary effects in Wang-Landau sampling. ${ }^{45}$ A sufficient overlap between different windows allowed swapping of configurations through a message passing interface (MPI) communication, thereby enhancing accuracy at the edges of the range sampled. The forces acting on the two colloids, projected on the vector connecting their centers were stored only in the late stages of the simulations when detailed balance is recovered. Overall a number of $\approx 10^{9}$ iterations were required to achieve satisfactory accuracy in the accumulated forces and weights. In the largest systems studied $(N=500)$, the orientational autocorrelation function of the vector along the end-to-end distance decays to zero through the algorithms used in this study in just $5 \times 10^{6}$ iterations. This fast sampling of configuration space, coupled with the systematic exploration of a range of values for the reaction coordinate through EXEDOS, provides a unique algorithm to extract the potential of mean force of colloids in solutions of long polymers.

The polymer-induced interactions on the two colloids are evaluated through the EXEDOS methodology using both the estimated weights to perform uniform sampling $[U$ $\left.=-k_{B} T \ln g(x)\right]$ and by integrating the mean projected forces as a function of separation. These two estimates provide knowledge of the potential of mean force to within a constant. As shown in Fig. 2 for two densities and $N=16$, by scaling the calculated weights from each of the ranges studied in parallel, excellent agreement is found between the PMFs obtained from the forces and the combined weights from different windows.

The simulation results are compared to the predictions of the PRISM-PY model with tangent-bead polymers. The monomer-colloid and colloid-colloid interactions are treated with the same potential as in Eq. (2). The polymer self structure factor $\omega_{p}(k)$ is obtained from the semiflexible chain model of Honnell et al. ${ }^{46}$ The persistence length of the chain is chosen to be $\xi_{p}=1.4$ which, along with $l / \sigma=1.12$, ac-

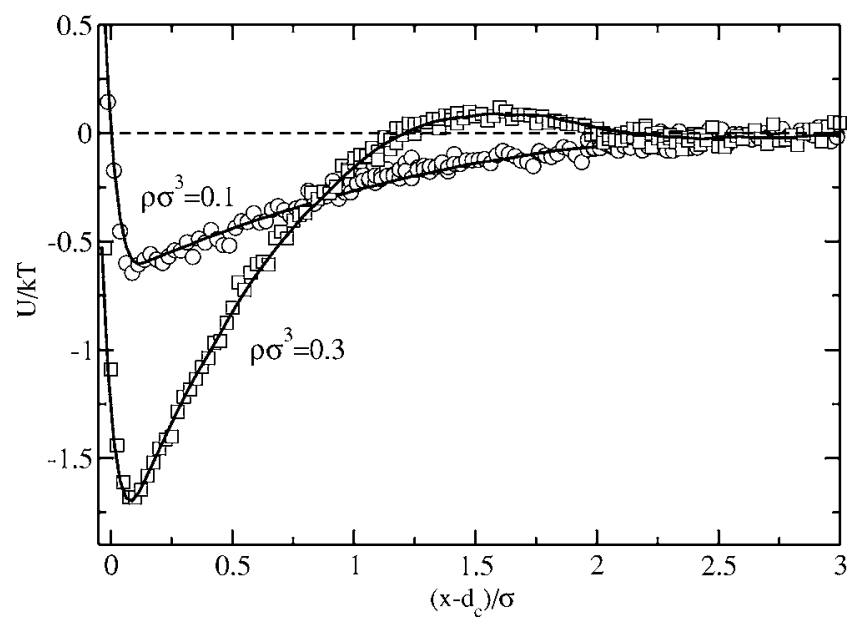

FIG. 2. Potential of mean force for two colloids immersed in a polymer solution as extracted from integration of the forces (continuous lines) and the weights required to achieve uniform sampling (open symbols). Three sets of weights from different windows are combined by scaling.

counts for chain flexibility and excluded volume through the average angle between any two connected segments, but does not account for excluded volume effects explicitly. Another approximation is that all sites on the chain are treated equally, thus neglecting effects due to chain ends.

\section{RESULTS}

\section{A. Depletion forces}

The most direct way to examine our results and compare to previous studies and theoretical calculations is through the depletion forces, which do not involve the scaling inherent to the potential of mean force. Figures 3(a) and 3(b) present the simulation predictions for two different chain lengths, $N$ $=16$ and $N=500$, in a dilute solution $\left(\rho \sigma^{3}=0.02\right)$. In the protein limit, it has been well established that the AsakuraOosawa model fails since it approximates polymers as phantom particles that do not interact with each other but act as hard particles of radius $R_{g}$ to the nanoparticles. Louis et al. ${ }^{31}$ proposed an alternative, semiempirical potential of the form $U(x) \sim(1-x / D)^{2}$, where $D$ is a constant that defines the range of the interaction. This form does not appear to be accurate for our systems, since it is based on a linear approximation of the depletion force. Such an approximation becomes increasingly inaccurate with chain size and systematically underestimates the PMF, an effect that can lead to second virial coefficients that increase with size ratio $q{ }^{33} \mathrm{~A}$ quadratic form for the forces seems to offer a better description, consistent from our earlier work on many-particle systems. $^{47}$

The results of PRISM-PY calculations are in qualitative agreement with the simulations; the dotted lines in Figs. 3(a) and 3(b) show the resulting forces extracted from calculations using an ideal structure factor $\omega(k)$. The model underestimates the depletion forces roughly by a factor of 1.8 for both systems, a feature recently reported for the melt regime. ${ }^{48}$ The discrepancies between theory and simulation could be due to several reasons. The theory incorporates a homogeneous self-structure for the polymer chain. In the vi- 

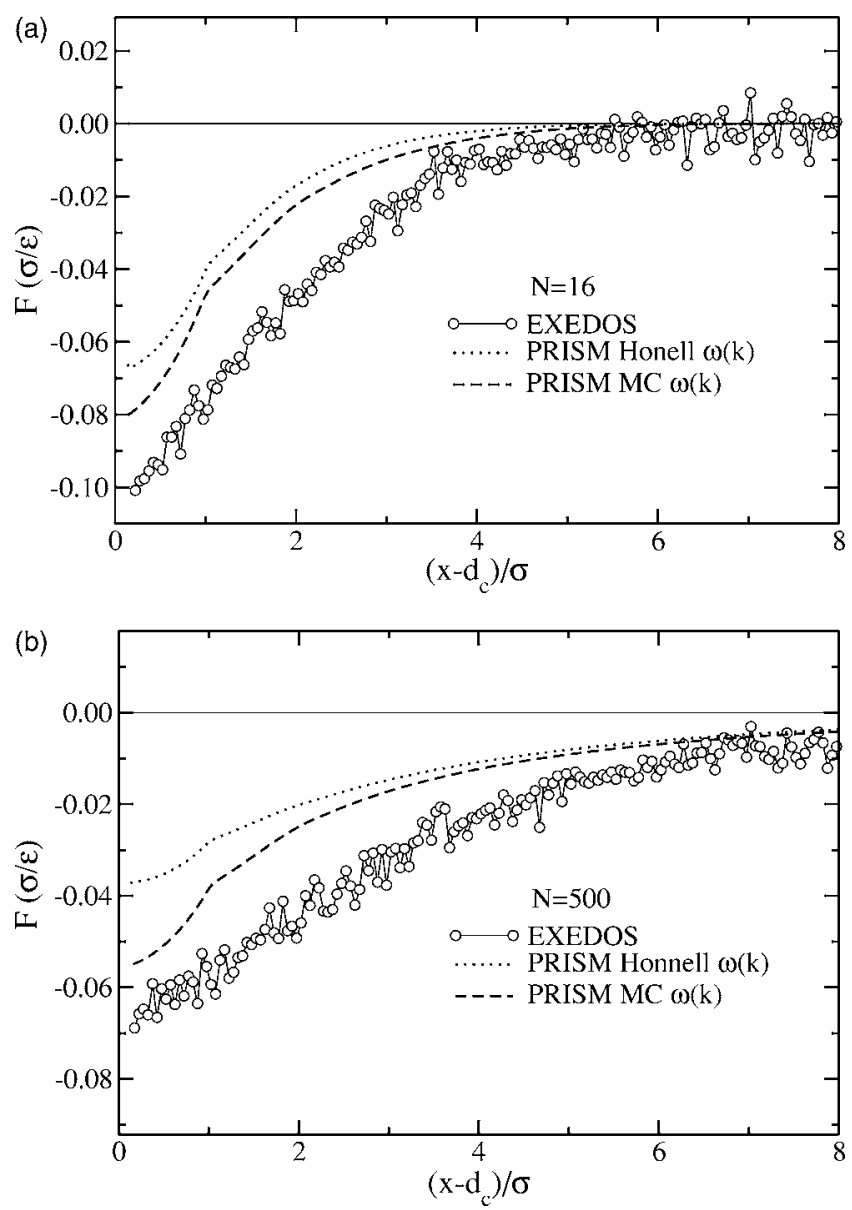

FIG. 3. Depletion forces between the two colloids as a function of the distance $x-d_{c}$ between the surfaces of the two spheres at $\rho \sigma^{3}=0.02$ for (a) $N=16$ and (b) $N=500$. Predictions from PRISM using the Honnell singlechain structure factor or the extracted from pure polymer MC simulations are also shown.

cinity of the colloids, however, the macromolecule can deform and reorient, resulting in significantly different configuration. ${ }^{34}$ The closure employed in this work does not consider nonlocal polymer-particle correlations; as recent predictions of PRISM with a modified Percus-Yevick closure for systems of ideal Gaussian threads (infinitely thin chains) and particles have shown, nonlocal correlations between polymer segments and particles lead to a stronger polymerinduced depletion attraction. ${ }^{19,20}$

In our previous simulations we have shown that the chain segments preferentially orient parallel to the particle surface; chain ends aggregate close to the colloid and the dimensions of the chains are altered. To quantify the importance of such effects, the intramolecular structure factors for pure polymer solutions at different densities can be determined directly from MC simulations and used as input for the polymer self-structure factor in PRISM. Figures 3(a) and 3(b) (dash lines) show that the PRISM predictions with the simulated polymer self-structure factor are in better quantitative agreement with simulation results. The strength of the depletion force is still underpredicted (approximately by a factor of 1.5), however, suggesting that deformations in the polymer structure due to the incorporation of the small colloid provide a major contribution to the induced depletion

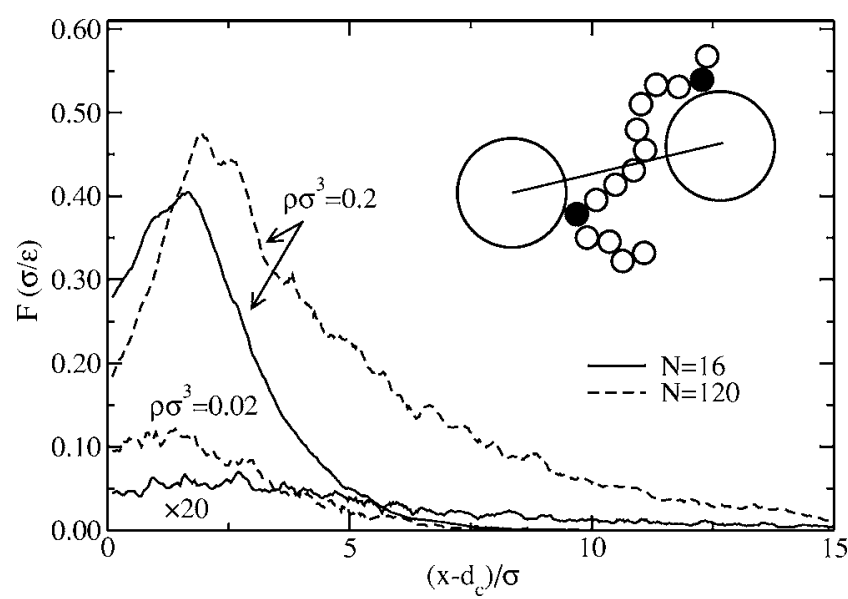

FIG. 4. Decomposition of the projected force into the part attributed to bridging chains. The forces at low density have been magnified for clarity.

potentials. In the limit of very small nanoparticles, the particles could also be affected by the inherent stiffness of the macromolecules.

We have further analyzed the induced forces on the particles by computing the part of the force (projected onto the separation vector) due to bridging chains, which are the molecules whose segments interact with both particles at the same time (see inset of Fig. 4). As the density increases $\left(\rho \sigma^{3}>0.2\right),{ }^{34}$ packing effects become significant, chains can no longer move freely from the region in between the particles, and the repulsive bridging forces increase. The values at contact decrease with increasing chain length at the same monomer density, since the particles more easily penetrate the longer chains. We have also found that the chain ends are preferentially found near the particle surface, extending the maximum range of the bridging forces up to the end-to-end distance of the polymers. ${ }^{34}$

\section{B. Depletion potentials}

Figure 5 shows the simulated potentials of mean force for systems with two different chain lengths, at the lowest density considered in this work. The potential of mean force was determined by integrating the projected forces. At such low densities, estimates for the PMFs from the weights can only be extracted using extreme values for the flatness criterion $(>95 \%)$ for the EXEDOS histograms, since the latter determines to a great extent the error with which the density of states is approximated. While the induced interactions are very weak with considerable statistical noise, it is evident that the PMFs are attractive with a range that extends to approximately $2.2 R_{g}$. PRISM-PY underestimates the magnitude of these induced interactions, as expected from the discussion on the depletion forces. In both systems, the value of the PMFs at contact $\left(x-d_{c}=0\right)$, as extracted with the Honnell structure factor, are underpredicted by approximately a factor of 2.5. Better quantitative agreement (by a factor of 1.7) is achieved by using the single-chain structure factor calculated from simulations of pure polymer solutions.

A novel, striking prediction of our simulations is that at ranges where the bridging forces become vanishingly small, a series of clear oscillations are found in the potential of 

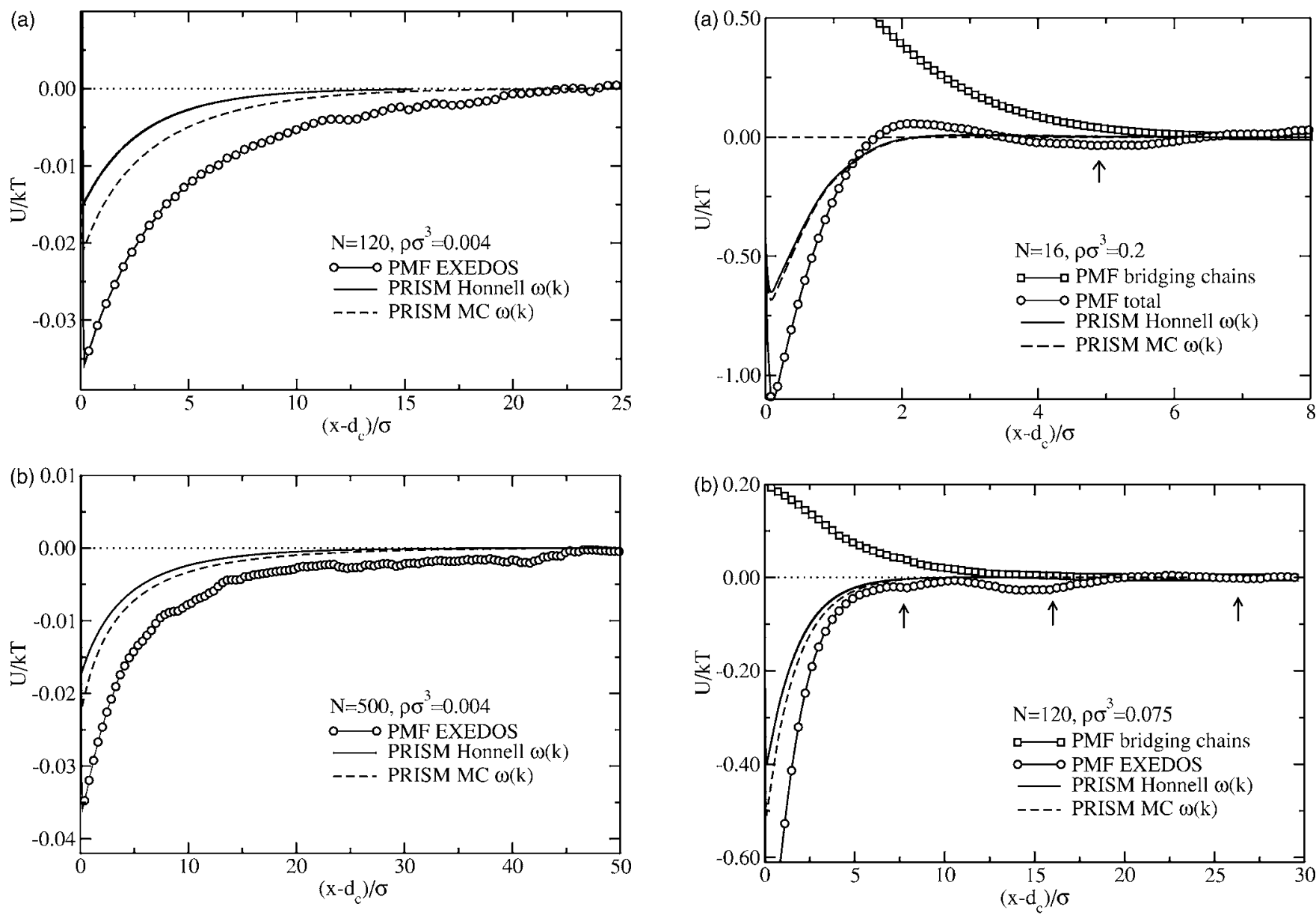

FIG. 5. Polymer-induced forces between the two colloids as a function of the distance $x-d_{c}$ between the two spheres at the low-density limit.

mean force. For systems of short chains, in the vicinity of the polymer overlap density $\left(N=16, \rho^{*} \sigma^{3} \approx 0.2\right)$, a well-defined minimum is found at approximately $2 R_{g}$ [Fig. 6(a)]. In these short-chain systems monomer packing effects emerge without the appearance of a clear semidilute regime. ${ }^{34}$ A repulsive peak is apparent, which arises from contributions of individual chains exerting a force on both colloids. For much longer systems, however, the polymer overlap concentration is considerably lower (e.g., for $N=120, \rho^{*} \sigma^{3} \approx 0.04$ ). Previous theoretical reports have assumed that the range of the potential of mean force is comparable to the size of the polymers in the dilute regime and to the correlation length $\xi$ in the semidilute regime. Simulation studies have generated PMFs that do not display any features apart from the attractive nature of the induced depletion interaction. Our simulations, however, suggest otherwise. Below and close to the overlap concentration, surprising oscillations are observed (even for systems having a low segment density).

Figure 6(b) displays the EXEDOS PMF at $\rho \sigma^{3}=0.075$ for $N=120$, i.e., near the polymer overlap density (in simulations $R_{g}$ decreases with density, resulting in a higher overlap concentration than that corresponding to the infinitedilution $R_{g}$ ). The minima that appear in the potential of mean force are highlighted by arrows (one at $R_{g}$, a second at $2 R_{g}$, and a third at $3 R_{g}$ ); they correspond to multiples of the actual $R_{g}$ at this density and cannot be correlated to polymer seg-

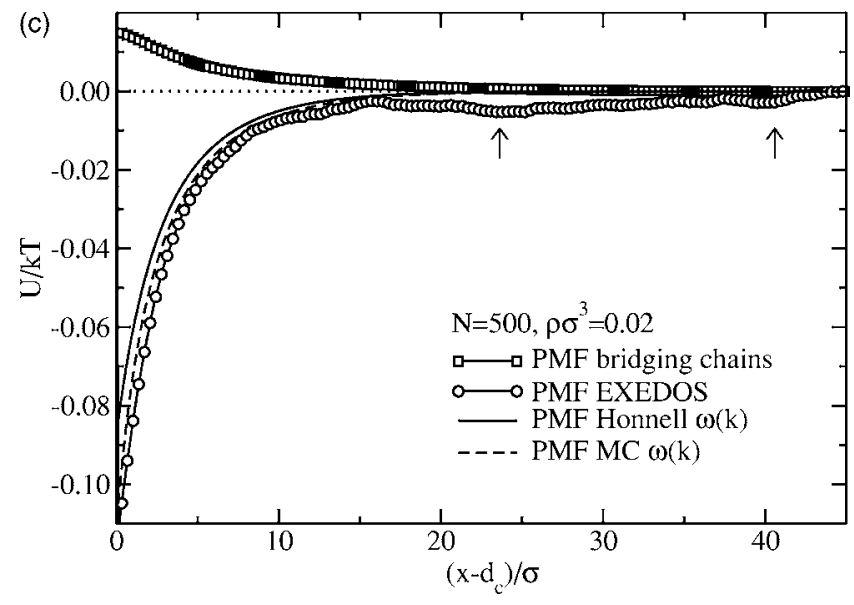

FIG. 6. Polymer-induced forces between the two colloids as a function of the distance $x-d_{c}$ between the two spheres in the proximity of the polymer overlap density.

ment packing effects (which were present for the $N=16$ case). These oscillations arise from polymer chains whose end segments are preferentially located in the vicinity of the particles. In our previous studies we have found that in the case of one particle in a polymer solution, chain end aggregation occurs at all densities. ${ }^{34}$ Figures $6(\mathrm{a})-6(\mathrm{c})$ present the integrated forces induced by the same chains on both particles at the same time (open squares). As supported by the range of the potential due to bridging chains, an arrangement where an individual long chain has its two ends positioned at 

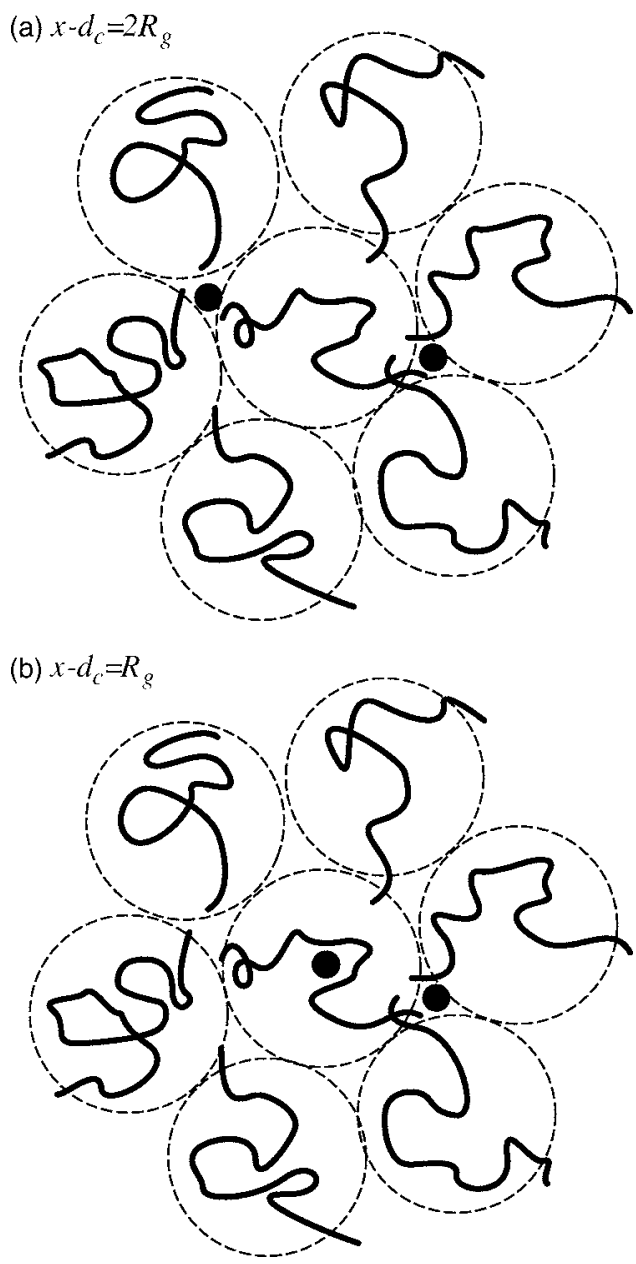

FIG. 7. Representative configurations with different separations between the two colloids, at the polymer overlap density, that induce localization of the particles.

the two particles is entropically favorable; work is required to overcome such a favorable arrangement and bring the two particles closer together. In a polymer solution, at the crossover from dilute to semidilute, more end segments of different chains can be in simultaneous contact with the particles when these are approximately $2 R_{g}$ apart. Such a configuration gives rise to a localization of the colloids, as shown schematically in Fig. 7(a); any tendency to decrease or increase their separation is entropically unfavorable.

A second, noteworthy feature of the PMF is that for sufficiently long chains additional minima arise. A clear minimum exist at both $R_{g}$ (first arrow) and $3 R_{g}$ (third arrow) in Fig. 6(b) for $N=120$. The presence of such a feature for long chains can be explained by considering an arrangement where one particle is incorporated into the polymer (chain wrapped around the colloid) and the second is located at the chain end [see Fig. 7(b)]. At higher densities $\left(\rho \sigma^{3}=0.1\right)$ the first minimum disappears and a repulsive peak develops up to $1 R_{g}$ for $N=120$, consistent with results shown in Fig. 6(a) for $N=16$. For even longer chains $(N=500)$ this feature persists over a comparable range of distances, but it is much broader [see first arrow in Fig. 6(c)]. In this case, the polymer can engulf the particle in a wider variety of configurations, thereby enlarging the range of separation where this

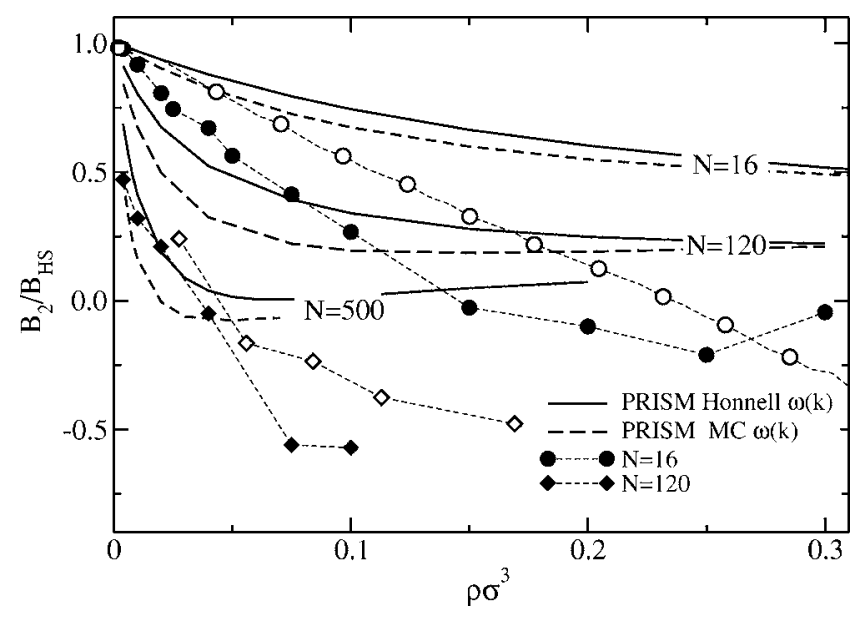

FIG. 8. Second virial coefficient normalized to the hard-sphere value. Estimates from PRISM using the Honnell and the MC single-chain structure factor are presented together with EXEDOS predictions. The open circles correspond to density-functional theory predictions (Ref. 50) and the open diamonds are the results from lattice simulations. ${ }^{33}$

favorable arrangement occurs. Such features indicate that high-precision calculations of the PMF require sampling over long distances (at least up to the end-to-end distance of the polymer chain) due to the oscillations and the scaling involved. For $N=500$ a quantitative prediction of the depletion interactions in the semidilute regime would involve systems with more than $\approx 10^{5}$ segments, which is outside the range of our computational capabilities.

Interestingly, a decade ago Semenov ${ }^{49}$ predicted (on the basis of mean-field arguments) the existence of polymerinduced long-range interactions in polymer solutions confined between solid walls by accounting for the effects of chain ends. This effect, to our knowledge, has not been fully accounted for in theories for nanoparticle-polymer systems. In contrast with large particles or flat walls, nanoparticles have the ability to nucleate multiple chain ends isotropically in a small region of space. Furthermore, macromolecules can actually wrap around the particles, inducing additional features in the depletion force. Theoretical calculations with the PRISM model (with the MC structure factor) provide potentials of mean force that are in striking qualitative agreement with simulations, but quantitative discrepancies remain due to such chain end effects.

\section{Second virial coefficient}

To demonstrate the importance and relevance of deformation effects and long range oscillations in the PMF, we estimated the second virial coefficient $B_{2}$ (normalized to the hard-sphere case) for both simulations and theoretical calculations:

$$
B_{2}^{*}=B_{2} / B_{\mathrm{HS}}=1-\frac{3}{d_{c}^{3}} \int_{d_{c}}^{\infty} x^{2}[\exp (-U(x) / k T)-1] d x .
$$

Results are shown in Fig. 8 for the three chain lengths studied. For the shortest chain $(N=16)$, the EXEDOS results exhibit an increase at high densities due to packing effects. PRISM-PY also predicts an increase in $B_{2}^{*}$ due to monomer packing at high densities (not shown). For longer chain 
lengths $(N=120)$, similar qualitative behavior is found between PRISM-PY and simulations, with $B_{2}^{*}$ saturating at densities above the polymer overlap density. The quantitative discrepancies may be attributed to the lower PMF predicted by PRISM-PY. At the semidilute crossover, the simulations show that oscillations in the PMF emerge and lead to a substantial decrease of the second virial coefficient. These oscillations make an accurate estimation of $B_{2}$ particularly challenging, as very long-range effects must be captured.

To compare our results to those of additional theoretical calculations we include predictions of the density-functional theory for the second virial coefficient provided by Patel and Egorov $^{50}$ (open circles) for $N=20$ and $d_{c}=5 \sigma$. The agreement between simulation (for $N=16$ and $d_{c}=4.9 \sigma$ ) and theory for similar conditions is not quantitative, but it is better than that observed for PRISM-PY. The origin of this better agreement is likely due to an improved estimation of the polymer segment density profile between the two colloids, which is important for short chains. Predictions for longer chains were not provided in Ref. 50. Figure 8 compares our results for $q=3.6$ to the results from lattice simulations for $q=3.86$, shown as open diamonds $\left(R_{g}=19.3\right.$ and $\phi_{p}$ was multiplied by 0.664$).{ }^{33}$ Below the overlap density $\left(\rho^{*} \sigma^{3} \approx 0.04\right)$ agreement is good; however in the crossover to the semidilute regime, lower values of $B_{2}$ are predicted by our simulations, as expected from our discussion.

An important issue discussed in the literature is the dependence of $B_{2}$ on $q$. Data from lattice simulations suggest that in the semidilute regime $B_{2}$ is higher for $q=7.78$ than for $q=3.86 .{ }^{33}$ PRISM, even with the MC $\omega(k)$, predicts that the second virial coefficient always decreases with increasing $q$, as shown in Fig. 8. Our simulation results support this dependence of $B_{2}$; it is important to state, however, that the integral in Eq. (5) involves a quadratic term over distance, which magnifies the effect (and uncertainty) of any longrange interaction. Due to the statistical uncertainty of simulations and the presence of long-range oscillations (not seen in previous studies) we do not report values for $N=500$. Suffice it to say, however, that an error of a fraction of a percent in the PMF for long chains, at large separations, can lead to one order-of-magnitude difference in the estimated second virial coefficient. It is also important to emphasize that the presence of long-range features in the PMF suggests that using the second virial coefficient (without higher-order terms) is only justified for extremely dilute nanoparticle suspensions. This result could be of significance in the study of protein separation using polymer solutions. ${ }^{51}$

\section{CONCLUSIONS}

We conclude this paper by emphasizing that solutions of long nonadsorbing polymers and small colloids in the protein limit exhibit long-range interactions that can persist over distances that are several multiples of $R_{g}$. Our continuum simulations using advanced methods have revealed the existence of previously unobserved long-range oscillations in the potential of mean force between the particles. Such oscillations are traced back to the pronounced tendency of chain ends to be near particle surfaces. This effect should be most impor- tant near the crossover from the dilute to the semidilute regime. Deformation of the chain near particle surfaces and entropic effects due to chain ends increase the magnitude of the depletion attraction and could lead to highly attractive second virial coefficient. These effects give rise to significant localization of the nanoparticles and may lead to long-range particle structures, affecting the phase behavior of the suspension. The emergence of these structures could in principle be probed by rheological measurements. The range and the form of the polymer-induced nanoparticle interactions is such that many-body effects become important at extremely low colloid concentrations. The significance of these effects on the phase diagram of colloid-polymer systems remains to be investigated.

\section{ACKNOWLEDGMENTS}

This work was supported by the Semiconductor Research Corporation (SRC) and the National Science Foundation (NSF) NIRT program.

${ }^{1}$ S. Asakura and F. Oosawa, J. Chem. Phys. 22, 1255 (1954).

${ }^{2}$ S. Asakura and F. Oosawa, J. Polym. Sci. 33, 183 (1958).

${ }^{3}$ J. F. Joanny, L. Leibler, and P. G. D. Gennes, J. Polym. Sci., Polym. Phys. Ed. 17, 1073 (1979).

${ }^{4}$ T. Odjik, J. Chem. Phys. 106, 3402 (1996).

${ }^{5}$ T. Odjik, Macromolecules 29, 1842 (1996).

${ }^{6}$ T. Odjik, Langmuir 13, 3579 (1997).

${ }^{7}$ T. Odjik, Physica A 278, 347 (2000).

${ }^{8}$ H. M. Schaink and J. A. M. Smit, J. Chem. Phys. 107, 1004 (1997).

${ }^{9}$ A. Hanke, E. Eisenriegler, and S. Dietrich, Phys. Rev. E 59, 6853 (1999).

${ }^{10}$ E. Eisenriegler, J. Phys.: Condens. Matter 12, A227 (2000).

${ }^{11}$ E. Eisenriegler, J. Chem. Phys. 113, 5091 (2000).

${ }^{12}$ R. Tuinier, G. A. Vliegenhart, and H. N. W. Lekkerkerker, J. Chem. Phys. 113, 10768 (2000).

${ }^{13}$ R. Tuinier, H. N. W. Lekkerkerker, and D. G. A. L. Aarts, Phys. Rev. E 65, 060801 (2002).

${ }^{14}$ R. Tuinier, D. G. A. L. Aarts, H. H. Wensik, and H. N. W. Lekkerkerker, Phys. Chem. Chem. Phys. 5, 3707 (2003).

${ }^{15}$ R. P. Sear, Phys. Rev. E 56, 4463 (2002).

${ }^{16}$ R. P. Sear, Phys. Rev. E 65, 062401 (2002).

${ }^{17}$ A. Yethiraj, C. K. Hall, and R. Dickman, J. Colloid Interface Sci. 151, 102 (1992).

${ }^{18}$ A. Chatterjee and K. S. Schweizer, J. Chem. Phys. 109, 10464 (1998).

${ }^{19}$ M. Fuchs and K. S. Schweizer, Europhys. Lett. 51, 621 (2000).

${ }^{20}$ M. Fuchs and K. S. Schweizer, Phys. Rev. E 64, 021514 (2001).

${ }^{21}$ A. M. Kulkarni, A. P. Chatterjee, K. S. Schweizer, and C. F. Zukoski, J. Phys.: Condens. Matter 12, A301 (2000).

${ }^{22}$ S. A. Shah, Y. L. Chen, K. S. Schweizer, and C. F. Zukoski, J. Chem. Phys. 118, 3350 (2003).

${ }^{23}$ R. Verma, J. C. Crocker, T. C. Lubensky, and A. G. Yodh, Phys. Rev. Lett. 81, 4004 (1998).

${ }^{24}$ R. Verma, J. C. Crocker, T. C. Lubensky, and A. G. Yodh, Macromolecules 33, 177 (2000).

${ }^{25}$ R. J. Owen, J. C. Crocker, R. Verma, and A. G. Yodh, Phys. Rev. E 64, 011401 (2001).

${ }^{26}$ A. G. Yodh, K.-H. Lin, J. C. Crocker, A. D. Dinsmore, R. Verma, and P. D. Kaplan, Philos. Trans. R. Soc. London 359, 921 (2001).

${ }^{27}$ M. Triantafillou and R. D. Kamien, Phys. Rev. E 59, 5621 (1999).

${ }^{28}$ E. J. Meijer and D. Frenkel, J. Chem. Phys. 100, 6873 (1994).

${ }^{29}$ R. Dickman and A. Yethiraj, J. Chem. Phys. 100, 4683 (1994).

${ }^{30}$ P. G. Bolhuis and A. A. Louis, Macromolecules 35, 1860 (2002).

${ }^{31}$ A. A. Louis, P. G. Bolhuis, and E. J. Meijer, J. Chem. Phys. 117, 1893 (2002).

${ }^{32}$ P. G. Bolhuis, A. A. Louis, and J.-P. Hansen, Phys. Rev. Lett. 89, 128302 (2002).

${ }^{33}$ P. G. Bolhuis, E. J. Meijer, and A. A. Louis, Phys. Rev. Lett. 90, 068304 (2003).

${ }^{34}$ M. Doxastakis, Y.-L. Chen, O. Guzmán, and J. J. de Pablo, J. Chem. Phys. 120, 9335 (2004). 
${ }^{35}$ N. C. Karayiannis, V. G. Mavrantzas, and D. N. Theodorou, Phys. Rev. Lett. 88, 105503 (2002).

${ }^{36}$ B. J. Banaszak and J. J. de Pablo, J. Chem. Phys. 119, 2456 (2003).

${ }^{37}$ E. B. Kim, R. Faller, Q. Yan, N. L. Abbott, and J. J. de Pablo, J. Chem. Phys. 117, 7781 (2002).

${ }^{38}$ N. Rathore, T. A. Knotts IV, and J. J. de Pablo, J. Chem. Phys. 118, 4285 (2003).

${ }^{39}$ N. Rathore, T. A. Knotts IV, and J. J. de Pablo, J. Chem. Phys. 118, 9460 (2003).

${ }^{40}$ J. P. Hansen and I. R. McDonald, Theory of Simple Liquids (Academic, London, 1986).

${ }^{41}$ F. Wang and D. P. Landau, Phys. Rev. Lett. 86, 2050 (2001).

${ }^{42}$ N. Rathore, Q. Yan, and J. J. de Pablo, J. Chem. Phys. 120, 5781 (2003).
${ }^{43}$ P. de Gennes, Scaling Concepts in Polymer Physics (Cornell University Press, Ithaca, 1979).

${ }^{44}$ M. Doi and S. F. Edwards, The Theory of Polymer Dynamics (Oxford University Press, New York, 1988).

${ }^{45}$ B. J. Schulz, K. Binder, M. Müller, and D. P. Landau, Phys. Rev. E 67, 067102 (2003).

${ }^{46}$ K. Honnell, J. Curro, and K. Schweizer, Macromolecules 23, 496 (1990).

${ }^{47}$ O. Guzmán and J. J. de Pablo, J. Chem. Phys. 118, 2392 (2003).

${ }^{48}$ J. B. Hooper, K. S. Schweizer, R. K. T. G. Desai, and P. Keblinski, J. Chem. Phys. 121, 6986 (2004).

${ }^{49}$ A. N. Semenov, J. Phys. II 6, 1759 (1996).

${ }^{50}$ N. Patel and S. A. Egorov, J. Chem. Phys. 121, 4987 (2004).

${ }^{51}$ J. Jiang and J. M. Prausnitz, J. Phys. Chem. B 103, 5560 (1999). 